\title{
PREVALENCE OF THE METABOLIC SYNDROME AMONG PATIENTS WITH TYPE 2 DIABETES MELLITUS IN URBAN NORTH-CENTRAL NIGERIA
}

\author{
PUEPET FH', ULOKO A', AKOGU IY', ANIEKWENSI E'. \\ Department of Medicine \\ University of Jos, 'Bayero University Kano, Nigeria. \\ Diabetes Screening and Care Foundation, \\ Jos, Nigeria \\ *Corresponding Author \\ Dr. Fabian H. Puepet \\ Endocrinology Unit, \\ Department of Medicine, \\ Jos University Teaching Hospital \\ P.M.B. 2076. Jos, Nigeria. \\ E-mail: puepetfh@yahoo.co.uk
}

Parts of this work were presented at the $18^{\text {in }}$ Annual Meeting and Clinical Congress of the American Association of Clinical Endocrinologists in May 2009, Houston, Texas, USA.

\begin{abstract}
Background: In most people with glucose intolerance or type 2 diabetes mellitus (DM), there is a multiple set of risk factors that commonly appear together forming what is now known as the 'Metabolic Syndrome' (MS). This 'clustering' of metabolic abnormalities that occur in the same individual appear to confer substantial additional cardiovascular risk. There has been no report on the MS among patients with type 2 DM in urban North-central Nigeria.

Objective: The objective of this study was to determine the prevalence of the MS among urban North-central Nigerians with type 2 DM and to describe the frequency of the syndrome's components.

Methods: Six hundred and thirty four (634) patients with type 2 diabetes attending the out-patient clinic of the Diabetes Screening and Care Centre, Jos, Nigeria were screened for the MS. A brief history was obtained and their anthropometric indices and blood pressure were measured. Fasting venous plasma samples were analyzed for lipids. Early morning spot urine samples were obtained for estimation of microalbuminuria using the Bayer DCA 2000" machine. The diagnosis of MS was made based on the new International Diabetes Federation (IDF) definition.

Results: Of the 634 patients enrolled with mean (standard deviation/SD) age 54.2(9.1) years, 56\% were females. The prevalence of MS was $63.6 \%(74.5 \%$ in males and $54.9 \%$ in females, $p<0.05)$. The mean (SD) age of patients with MS was $54.7(9.5)$ years. About $80 \%$ of the patients were centrally obese, $63 \%$ had hypertension, $62 \%$ had high triglycerides and $70 \%$ with low HDL-Chol. Among patients with MS, $79 \%$ had dyslipidaemia, $41 \%$ had BMI e" 30 , and $36 \%$ had microalbuminuria.

Conclusion: The prevalence of the MS in this group of upland Nigerian patients with type 2 diabetes is moderately high. This probably reflects the adoption of Western lifestyles and diets that require intervention.
\end{abstract}

Keywords: Prevalence, Metabolic syndrome, Diabetes.

\section{INTRODUCTION}

Each year, 3.2 million people around the world die from complications associated with diabetes. In countries with a high diabetes incidence, such as in the Pacific and the Middle East, as many as one in four deaths in adults aged between 35 and 64 years is due to the disease (1). Type 2 diabetes mellitus (DM), which accounts for $90 \%$ of all diabetes, has become one of the major causes of premature illness and death, mainly through increased risk of cardiovascular diseases (CVDs) which is responsible for up to $80 \%$ of these deaths (2). In most people with glucose intolerance or type $2 \mathrm{DM}$, there is a multiple set of risk factors that commonly appear together, forming what is now known as the 'Metabolic Syndrome' (MS). This clustering of metabolic abnormalities that occur in the same individual appear to confer a substantial additional cardiovascular risk over and above the sum of the risk associated with each abnormality $(3,4)$. MS is a cluster of the most dangerous heart attack risk factors: diabetes and raised fasting plasma glucose, abdominal obesity, high cholesterol and high blood pressure $(6,7)$. The underlying cause of MS continues to challenge the experts but both insulin resistance and central obesity are considered significant factors $(8,9)$. Obesity is associated with insulin resistance and the MS. Central or abdominal obesity contributes to hypertension, high serum cholesterol, low high density lipoprotein (HDL)-cholesterol, high triglycerides and hyperglycaemia and is independently associated with higher CVD risk (9-11).

It is estimated that around $20-25 \%$ of the world's adult population have the MS and they are twice as likely to have a heart attack or stroke compared with people without the syndrome $(7,12)$. The more the components of the metabolic syndrome that are evident, the higher is the cardiovascular metabolic rate (8). There is paucity of reports on the metabolic syndrome in developing countries including Nigeria. The prevalence rates of the metabolic syndrome among patients with type 2 diabetes in Iraq (13) and Saudi Arabia (14) are 86\% and $56 \%$ respectively. The MS was seen in $51 \%$ of South-Western Nigerians (15). There has been no report on the MS from Jos, North-central Nigeria.

The objective of this study was to determine the prevalence of the MS among urban North-central Nigerians with type 2 diabetes and to describe the frequency of the syndrome's components. 


\section{PATIENTS AND METHODS}

Six hundred and thirty four (634) patients with type 2 diabetes attending the out-patient clinic of the Diabetes Screening and Care Centre, Jos, between January 2006 and December 2008 were screened for the MS. Patients known to have had type $2 \mathrm{DM}$ for at least 6 months, and aged 30-80 years (most of whom were middle and upper cadre civil servants and business men/women) were consecutively enrolled after informed consent was obtained. All study subjects were residents of Jos metropolis and environs. The WHO Consultation and Expert Committee on the diagnosis, classification of DM criteria (5) was used for the diagnosis and classification of type 2 DM. A brief clinical history was obtained and their anthropometric indices and blood pressures were measured. Fasting venous plasma samples were analyzed for lipids. Microalbuminuria was quantitatively estimated from early morning spot urine samples using the Bayer DCA 2000* machine. Information obtained was recorded in a pretested questionnaire prepared for the purpose of the study. The diagnosis of the MS was made based on the new IDF definition $(6,7)$, Central obesity (waist circumference, [WC] e" $94 \mathrm{~cm}$ in males and e" $80 \mathrm{~cm}$ in females) plus two of the four factors: raised triglycerides $(\mathrm{e} " 1.7 \mathrm{mmol} / \mathrm{l})$, reduced HDL-Chol $(<1.03 \mathrm{mmol} / 1$ in males and $<1.29 \mathrm{mmol} / 1$ in females), raised BP (systolic BPe"130 $\mathrm{mmHg}$, or diastolic BPe" $85 \mathrm{mmHg}$ or treatment of previously diagnosed hypertension), raised FPG (e" $5.6 \mathrm{mmol} / 1$ or previously diagnosed type 2 diabetes). Statistics: Data analysis was carried out using EPI-INFO (2002) statistical programme. Means $( \pm \mathrm{SD})$ were calculated for quantitative data, and proportions for categorical variables. The student's $-t$ test was used for comparisons; $p$-values $<0.05$ were considered significant.

Ethics: Ethical approval was obtained from the Research and Ethical Committee of the Jos University Teaching Hospital, Plateau State and consent was obtained from each patient before the commencement of the study.

\section{RESULTS}

Of the 634 patients enrolled with mean (SD) age $54.2(9.1)$ years, $355(56 \%)$ were females and 279 (44\%) were males. There was no significant difference in the mean (SD) ages of males, 54.5 (9.5) years and females, 53.7 (10.1) years, $p>0.05$. The MS was seen in 403 patients, $63.6 \%$ (51.6\% males, $48.4 \%$ females). The prevalence rates of MS in males and females were $74.5 \%$ and $54.9 \%$ respectively, being significantly higher in males than in females $(p<0.05)$. The mean (SD) age of patients with MS was 54.7 (9.5) years. Figure 1 shows the prevalence rates of the components of MS determined. About $80 \%$ of the study population was centrally obese using WC, $63.1 \%$ with hypertension, $62 \%$ with high triglycerides and $70 \%$ with low HDL-Cholesterol. The prevalence of dyslipidaemia in the patients with MS was $78.9 \%$, the rates were $59.1 \%$ and $43.1 \%$ in males and females respectively $(p<0.05)$. The mean (SD) BMI of patients with MS was 29.8(4.4) $\mathrm{kg} / \mathrm{m}^{2}$. Only $41.4 \%$ of patients with the MS had BMI e" $30 \mathrm{~kg} / \mathrm{m}^{2}$. Microalbuminuria was detected in $34.6 \%$ of patients with the MS. The anthropometric and biochemical characteristics of the Patients with MS are summarized in table 1.

Figure 1: Components of Metabolic Syndrome

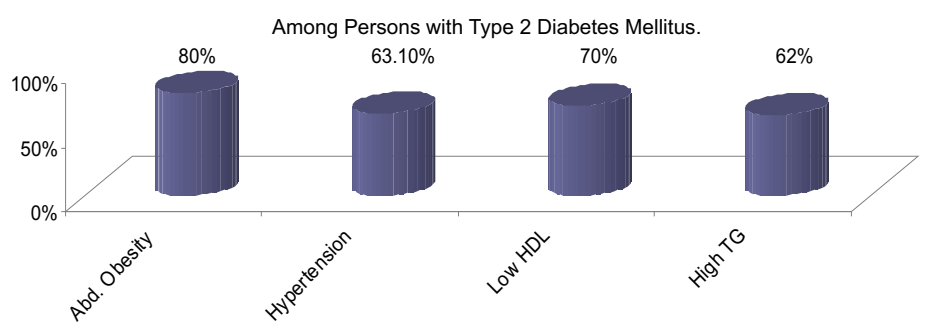

Table 1: Anthropometric and Biochemical Features of Patients with the Metabolic Syndrome.

\begin{tabular}{ll}
\hline $\mathbf{B M I}\left(\mathbf{K g} / \mathbf{m}^{2}\right)$ & Freq. $(\%)$ \\
\hline $18.5-24.9$ & $38(9.4 \%)$ \\
$25.0-29.9$ & $198(49.1 \%)$ \\
e”30 & $167(41.4 \%)$ \\
WC $(\mathrm{cm})$ & Mean (SD) \\
Males & $98.6(3.8)$ \\
Females & $91.3(10.1)$ \\
Microalbuminuria & Freq. $(\%)$ \\
(5-300mg/L) & $139(34.6 \%)$ \\
Dyslipidaemia & \\
Males & \\
Females & $165(59.1 \%)$ \\
\hline
\end{tabular}

\section{DISCUSSION}

Changing lifestyles, characterized by increase caloric intake and reduction in physical activity coupled with westernization have lead to an increase in prevalence of diabetes in the developing countries including Nigeria (6).

The World Health Organization (WHO) criteria for the MS (15) are different from those of the IDF $(6,7)$. The WHO definition uses the BMI and includes microalbuminuria, which is often difficult to measure in tropical countries. Although, microalbuminuria was determined in our study, we used the IDF definition for MS. In this study, we found a high prevalence of the MS (63.6\%) in North-Central Nigeria. In comparison, MS was seen in $51 \%$ of South-Western Nigerians with type 2 diabetes, where the WHO criteria were used (15). In the south western Nigerian study, the ratio of females to males with MS was $6: 4$ which is a reverse of the observation in this study, the use of different diagnostic criteria for MS may account for the difference. However, North-central Nigerian women have been known to be more active than the men. In Iraqi (NIH-ATP Panel III criteria) (9) and Saudi (WHO criteria) (14) patients with type 2 diabetes, the prevalence rates of MS were $86 \%$ and $56 \%$ respectively and the commonest component in both countries was hypertension. In this study, the commonest component of MS was abdominal obesity. The prevalence rates of the MS and its components are strongly dependent on the definition of the different components of the syndrome, which are still not accepted globally. A possible explanation of the high rates of MS in Nigerian patients is the adoption of Western lifestyle (increased caloric intake and reduction in physical activity).

The prevalence of the MS in this group of upland Nigerian patients with type 2 diabetes is high. This high figure is of great concern and probably reflects the adoption of Western lifestyles and dietary changes that require intervention. Further study needs to be carried out in rural Plateau State to throw more light on the aetiopathogenesis of the MS in this group of Nigerians.

\section{REFERENCES}

1. Diabetes Atlas, $2^{\text {nd }}$ edition, International Diabetes Federation, 2003.

2. UKPDS Group. UK Prospective Diabetes Study 17: A nine year update of a randomized, controlled trial on the effect of improved metabolic control on complications in NIDDM. Ann Intern Med, 1996; 124: 136-145. 
3. Sattar N, Gaw A, Scherbakova O. Metabolic syndrome with and without $\mathrm{C}$-reactive protein as a predictor of coronary heart disease and diabetes in the West of Scotland coronary prevention study. Circulation, 2003; 108: 414-419.

4. Golden SH, Folson AR, Coresh J. Risk factor grouping related to insulin resistance and their synergistic effects on subclinical atherosclerosis: the atherosclerosis risk in communities study. Diabetes, 2002; 51: 3069-3076.

5. Alberti KGM, Zimmet PZ for WHO Consultation. Definition, diagnosis and classification of diabetes mellitus: Provisional report of a WHO Consultation. Diabet Med, 1998; 15: 539553.

6. Alberti KG, Zimmet P, Shaw J. Metabolic syndrome- a new world-wide definition. A consensus statement from the International Diabetes Federation. Diabet Med, 2006; 23(5):469-480.

7. www.idf.org/metabolic syndrome, website of the International Diabetes Federation.

8. $\mathrm{Hu} \mathrm{G}$, Qiao Q, Tuomilehto $\mathrm{J}$ et al. Plasma insulin and cardiovascular mortality in non-diabetic European men and women: a meta-analysis of Data from eleven prospective studies. The DECODE Insulin Study Group. Diabetologia, 2004; 47: 1245-1256.
9. Carr DB, Utzschneider KM, Hull RL et al. Intra-abdominal fat is a major determinant of the National Cholesterol Education Program Adult Treatment Panel III Criteria for metabolic syndrome. Diabetes, 2004; 53(8): 2087-2094.

10. Aderson PJ, Critchley JAJH, Chan JCN. Factor analysis of the metabolic syndrome: Obesity Vs Insulin resistance as the central abnormality. International Journal of Obesity, 2001; 25: 1782.

11. Zimmet P, Alberti KGMM, Shaw J. Global and societal implications of the diabetes epidemic. Nature, 2001; 414: 782-787.

12. Stern M, Williams K, Gonzalez-Villapando C. Does the metabolic syndrome improve identification of individuals at risk of type 2 diabetes and/or cardiovascular disease? Diabetes Care, 2004; 27(11): 2676-2681.

13. Mansour AA. Prevalence of the metabolic syndrome among patients with type 2 diabetes in Iraq. Diabetes Int, 2007; 15(1): 12.

14. Akbar DH. Metabolic syndrome is common in Saudi type 2 diabetic patients. Diabetes Int, 2002; 12:47-49.

15. Adediran OS, Edo AE, Jimoh AK, Ohwovoriole AE. Prevalence of the metabolic syndrome among Nigerians with type 2 diabetes. Diabetes Int, 2007; 15(1): 13-14. 